\title{
Lung cancer gene associated with COPD: triple whammy or possible confounding effect?
}

\author{
R.P. Young*, , R.J. Hopkins*, B.A. Hay*, M.J. Epton ", P.N. Black* and G.D. Gamble*
}

ABSTRACT: Recently, several large genome-wide association studies have identified a putative "lung cancer" locus in the nicotinic acetylcholine receptor subunit genes (nAChR) on 15q25. However, these findings may be confounded by the presence of chronic obstructive pulmonary disease (COPD), which is also strongly associated with smoking exposure and lung cancer. This is likely as the prevalence of COPD in lung cancer cohorts is as much as two-fold greater than that reported in smoking control populations (50 versus $20 \%$ ).

The present authors compared the genotype frequencies of the most strongly associated single nucleotide polymorphism (rs16969968) in the $\alpha 5$ subunit of the nAChR gene cluster between three matched smoking cohorts.

The AA genotype was found to be more frequent and was seen in 437 (16\%) lung cancer cases and 445 (14\%) COPD cases compared with 475 (9\%) healthy smoking controls. More importantly, when 429 lung cancer cases were divided according to spirometry results (performed within 3 months of diagnosis, prior to surgery and in the absence of effusions or collapse), the AA genotype was present in 19 and $11 \%$ of cases with and without COPD, respectively.

These findings suggest that the association between the $\alpha 5$ subunit nicotinic acetylcholine receptor single nucleotide polymorphism and lung cancer may, in part, be confounded by chronic obstructive pulmonary disease.

KEYWORDS: Association study, chronic obstructive pulmonary disease, confounding, lung cancer, nicotinic acetylcholine receptor, polymorphism

$\mathbf{R}$ ecent genome-wide association studies (GWAS) [1-3] have identified a putative lung cancer locus in the nicotinic acetylcholine receptor (nAChR) subunit genes on 15q25. Some debate exists as to whether single nucleotide polymorphisms (SNPs) in this region are associated with lung cancer, nicotine addiction or both. A large study by THORGEIRSSON et al. [1] reports that most of the effect relates to nicotine addiction, with only a small effect on lung cancer risk and an even smaller effect on peripheral arterial disease (PAD) risk. In identifying this "nicotine addiction" gene, THORGEIRSSON et al. [1] replicated the results of a well-designed candidate gene study reported in 2007 of 1,800 smokers and ex-smokers [4].

The GWAS described previously did not consider that the results may be confounded by the presence of chronic obstructive pulmonary disease (COPD), characterised by reduced lung function, which is also strongly associated with smoking exposure, lung cancer, lung cancer mortality and peripheral arterial disease [5-15]. Population studies show that COPD, defined by the presence of air flow limitation on spirometry (primarily reduced forced expiratory volume in one second; FEV1), is consistently under-recognised in the community, with as much as $50-80 \%$ of cases remaining undiagnosed $[5,16,17]$. This is due to the insidious decline in lung function in COPD and the under-utilisation of spirometry to screen those with a smoking history [10]. Smokers with reduced FEV1 carry as much as a five- to six-fold risk of lung cancer compared to smokers with normal lung function [11]. One prospective study has shown that reduced FEV1 is the most important risk factor for lung cancer, even more important than that conferred by age and pack-yr history [9]. Several studies have shown that $50-70 \%$ of patients diagnosed with lung cancer have spirometric evidence of COPD (based on Global Initiative of Obstructive Lung Disease (GOLD) criteria) [18-21]. However, studies of heavy smokers, who contribute to the vast majority of controls in the GWAS, report that

\section{AFFILIATIONS}

*Dept of Medicine, University of Auckland,

\#Synergenz Biosciences Ltd, Auckland, and

"Dept of Medicine, University of Otago, Christchurch, New Zealand.

CORRESPONDENCE

R.P. Young

Dept of Medicine

Auckland Hospital

Private Bag 92019

Auckland

New Zealand

Fax: 6496236456

E-mail: roberty@adhb.govt.nz

Received:

June 192008

Accepted after revision:

August 182008

STATEMENT OF INTEREST

A statement of interest for R.P. Young and for the study itself can be found

at www.erj.ersjournals.com/misc/

statements.shtml 
20-30\% have COPD based on the same spirometric criteria $[6-8,16]$. Similar findings are reported in studies of smokers admitted to hospital with a variety of acute medical problems [17].

As the prevalence of COPD may be as much as two-fold greater in lung cancer cases compared with smoking controls, it is possible that any associations reported by these lung cancer GWAS may be confounded by an association with COPD. That is, the genetic variant putatively associated with lung cancer may in fact be associated with COPD and not lung cancer due to the difference in frequency of COPD between lung cancer cases and controls. Where genetic variants are found to be independently associated with both lung cancer and COPD, it is possible that these variants confer susceptibility to both through overlapping pathogenic pathways, such as those underlying smoking-induced inflammation [22]. By comparing the frequency of the candidate polymorphisms, either between patients with COPD and lung cancer, or within lung cancer cohorts in which lung function has been recorded and coexisting COPD identified, these two possibilities can be distinguished. Using both these approaches, the recently reported GWAS lung cancer gene association study has been re-assessed and the results presented herein.

\section{MATERIALS AND METHODS \\ Study subjects}

The present authors recruited people of Caucasian ancestry based on their grandparents' descent (all four grandparents of Caucasian descent). Patients with lung cancer were identified through hospital records and specialist clinics between 2004 and 2007. Lung cancer cases were aged $>40$ yrs and the diagnosis was confirmed through histological or cytological specimens in $95 \%$ of cases. Nonsmokers with lung cancer were excluded from the study and only primary lung cancer cases with the following pathological diagnosis were included: adenocarcinoma, squamous cell cancer, small cell cancer and nonsmall cell cancer (generally large cell or bronchoalveolar subtypes). Spirometry in the lung cancer cases was performed using American Thoracic Society (ATS) criteria within 3 months of lung cancer diagnosis, prior to surgery and in the absence of pleural effusions or lung collapse on plain chest radiographs. For lung cancer cases that had already undergone surgery, pre-operative lung function performed by the hospital lung function laboratory (using ATS criteria) was sourced from medical records.

Patients with COPD were identified through hospital specialist clinics between 2003 and 2007. Subjects recruited into the study were aged $>40 \mathrm{yrs}$, with a minimum smoking history of 15 pack-yrs and COPD confirmed by a respiratory specialist based on spirometric criteria.

Control subjects were recruited based on the following criteria: aged $45-80$ yrs and with a minimum smoking history of 15 pack-yrs. Control subjects were volunteers who were identified through either a community postal advert or while attending community-based retired military/servicemen's clubs located in the same patient catchment as those serving the lung cancer and COPD hospital clinics. All participants gave written informed consent, and underwent blood sampling for DNA extraction, spirometry and an investigator-administered questionnaire. Controls with COPD (GOLD stage I or more) were excluded from further analysis.

Spirometry was performed using a portable spirometer (EasyOne $^{\mathrm{TM}}$; ndd Medizintechnik AG, Zurich, Switzerland). A modified ATS respiratory questionnaire was administered to all cases and controls, which collected data on demographic variables such as age, sex, medical history, family history of lung disease, active and passive tobacco exposure, respiratory symptoms and occupational aero-pollutant exposures. The study was approved by the Local Ethics Committee (Auckland, New Zealand).

\section{Study design}

The present retrospective case-control study compared smokers of the same ethnicity and comparable demographic variables (specifically age, sex and pack-yr smoking history). However, in contrast to the majority of case-control studies, the controls in the current study were carefully chosen to be representative of the majority of smokers who have maintained normal or near-normal lung function (estimated to be $80 \%$ by excluding those with GOLD stage II COPD) despite decades of smoking [6-8, 14]. Accordingly, such a group best reflects those smokers least likely to get lung cancer, thus minimising phenotype misclassification and improving the power to detect differences between affected and unaffected smokers [23].

\section{Methods}

Genomic DNA was extracted from whole blood samples using standard salt-based methods. Purified genomic DNA was aliquoted (10 ng. $\mu \mathrm{L}^{-1}$ concentration) into 96-well plates and genotyped on a Sequenom ${ }^{\mathrm{TM}}$ Autoflex Mass Spectrometer (Sequenom, San Diego, CA, USA) and a Samsung 24-pin nanodispenser (Samsung, Suwon City, Korea) by the Australian Genome Research Facility using sequences designed in-house (table 1), and amplification and separation methods (iPLEX ${ }^{\mathrm{TM}}$; Sequenom) described herein. The conditions used for the PCR multiplex reaction and the final concentrations were as follows. Buffer: $15 \mathrm{mM} \mathrm{MgCl}_{2} 1.25 \times$, 25 mM MgCl 1.625 mM; dNTP mix: 25 mM $500 \mu \mathrm{M}$; primers: $4 \mu \mathrm{M} 100 \mathrm{Nm}$; Taq polymerase (Qiagen hot start; Qiagen $\mathrm{GmbH}$, Hilden, Germany) $0.15 \mathrm{U} \cdot$ reaction $^{-1}$; genomic DNA: $10 \mathrm{ng} \cdot \mu \mathrm{L}^{-1}$. Cycling times were $95^{\circ} \mathrm{C}$ for $15 \mathrm{~min}$, followed by $95^{\circ} \mathrm{C}$ for $15 \mathrm{~s}, 56^{\circ} \mathrm{C}$ for $30 \mathrm{~s}$ and $72^{\circ} \mathrm{C}$ for $30 \mathrm{~s}$ for 45 cycles, with a prolonged extension time for $3 \mathrm{~min}$ at $72^{\circ} \mathrm{C}$ to finish. Shrimp alkaline phosphatase (SAP) treatment $(2-5 \mu \mathrm{L}$ per PCR reaction) was used as an incubator at $35^{\circ} \mathrm{C}$ for $30 \mathrm{~min}$, followed by an extension reaction $(2-7 \mu \mathrm{L}$ added after SAP treatment) with the following volumes per reaction: water, $0.76 \mu \mathrm{L}$; homologous mass extend (hME) $10 \times$ termination buffer, $0.2 \mu \mathrm{L}$; hME primer $(10 \mu \mathrm{M}), 1 \mu \mathrm{L}$; MassEXTEND enzyme (Sequenom), $0.04 \mu \mathrm{L}$. The extension assay was terminated after single base extension and allelic separation using a matrix-assisted laser desorption/ionisation time-of-flight mass spectrometer, which allowed genotypes for each SNP to be determined. The present study compared the genotype frequencies of the two most strongly associated SNPs (rs16969968 and rs1051730) in tight linkage disequilibrium from the $\alpha 5$ and $\alpha 3$ subunits, respectively, of the nAChR gene cluster with call rates of $\geqslant 96 \%$ for each SNP in each cohort. 


\begin{tabular}{|c|c|c|c|}
\hline TABLE 1 & $\begin{array}{l}\text { Primer sequences for iPLEX } \\
\text { nucleotide polymorphism (S }\end{array}$ & ssay for the nicotinic a & tor $\alpha 5$ and $\alpha 3$ subunit single \\
\hline SNP ID & First PCR primer & Second PCR primer & Extension primer \\
\hline \multirow[t]{2}{*}{ rs16969968 } & ACGTTGGATGCACGG & ACGTTGGATGTCTAGAAA & CATTGGAAGCTGCGCTC \\
\hline & ACATCATTTTCCTTC & CACATTGGAAGC & \\
\hline rs1051730 & ACGTTGGATGCAGCA & ACGTTGGATGTCAAGGAC & TCATCAAAGCCCCAGGCTA \\
\hline
\end{tabular}

$\mathrm{iPLEX}^{\mathrm{TM}}$ is manufactured by Sequenom, San Diego, CA, USA.

\section{Analysis}

Patient characteristics in the cases and controls were compared by ANOVA for continuous variables and Chi-squared test for discrete variables (Mantel-Haenszel, odds ratio (OR)). Genotype and allele frequencies were checked for each SNP by Hardy-Weinberg Equilibrium (HWE). Population admixture across cohorts was performed using structure analysis on genotyping data from 40 unrelated SNPs [24]. Distortions in the genotype frequencies were identified between cases and controls using two-by-two contingency tables.

\section{RESULTS}

\section{Demographic variables and genotyping}

Characteristics of the lung cancer cases, COPD cases and healthy control smokers are summarised in table 2. The demographic variables and histological subtypes of the lung cancer cases are comparable to a recently published series [25]. In the present study, staging at diagnosis was also comparable to the previously published series (data not shown) suggesting the current lung cancer cohort was representative. Although the COPD cases have higher pack-yr exposure than the lung cancer cases and healthy control smokers $(p<0.05)$, this is no longer the case after log transformation of pack-yrs (data not shown) and reflects outliers with high smoking histories in the COPD cohort. All cohorts were comparable with respect to age started smoking, years smoked, years since quitting and cigarettes smoked per day (table 2). Overall, the present authors believe the three groups have comparable smoking exposure and the pack-yr difference is of no clinical significance. The lower frequency of current smokers in the lung cancer and COPD cohorts compared with healthy smokers (35 versus 40 versus $48 \%$, respectively) may reflect the presence of COPD leading to higher quit rates. The lung cancer cases, COPD cases and smoking controls were also comparable with respect to other aero-pollutant exposures, although the lung cancer and COPD cohorts had higher asbestos exposure (23 versus 22 versus 16\%, respectively), slightly higher dust exposure (63 versus 59 versus 47\%, respectively) but comparable fume exposure (41 versus 40 versus $38 \%$, respectively). The lung cancer cohort also had higher rates of family history of lung cancer compared with the COPD cases and healthy smokers (19 versus 11 versus 9\%), whereas family history of COPD was marginally higher in those with COPD (33 versus 37 versus $28 \%$ ). As expected, lung function was worse in the lung cancer and COPD cohorts compared with the healthy smoker controls. Testing lung function in the lung cancer cases (as described previously) allowed the present authors to test for confounding by COPD. Genotype frequencies for the $\alpha 5$ nAChR G/A SNP (rs16969968) are shown in table 3. The genotype frequencies for the $\alpha 3 \mathrm{nAChR}$ C/T SNP (rs1051730) were virtually identical to those of the rs16969968 SNP (where CC and GG are homozygous for the major allele, while TT and AA are homozygous for the minor allele). The observed genotypes for the two SNPs in the current study were $>99 \%$ concordant (as expected given tight linkage disequilibrium between them) and, in HWE, significant genotyping error could be excluded. No evidence was found for population stratification between the cohorts using 40 unlinked SNPs from unrelated genes (mean Chi-squared $=3.3, p=0.58$ ). The genotype frequencies were comparable to those reported in the literature and from the International Hapmap Project [26].

\section{TABLE 2 Study characteristics}

\begin{tabular}{|c|c|c|c|}
\hline Parameter & Lung cancer & COPD & Control smokers \\
\hline Subjects $n$ & 454 & 458 & 488 \\
\hline Males & 53 & 59 & 60 \\
\hline Age yrs & $69 \pm 10$ & $66 \pm 9$ & $65 \pm 10$ \\
\hline \multicolumn{4}{|l|}{ Smoking history } \\
\hline Current smoker & 35 & 40 & 48 \\
\hline Age started smoking yrs & $18 \pm 4$ & $17 \pm 3$ & $17 \pm 3$ \\
\hline Length of time smoked yrs & $41 \pm 12$ & $42 \pm 11$ & $35 \pm 11$ \\
\hline Pack-yrs & $41 \pm 25^{*}$ & $47 \pm 20^{*}$ & $40 \pm 19^{*}$ \\
\hline Cigarettes $\cdot$ day $^{-1}$ & $20 \pm 10$ & $23 \pm 9$ & $24 \pm 11$ \\
\hline Time since quitting yrs & $11.4 \pm 6.7$ & $9.8 \pm 7.4$ & $13.9 \pm 8.1$ \\
\hline \multicolumn{4}{|l|}{ History of other exposures } \\
\hline Work dust & $63^{*}$ & $59^{*}$ & $47^{\star}$ \\
\hline Work fume & 41 & 40 & 38 \\
\hline Asbestos & $23^{*}$ & $22^{*}$ & $16^{*}$ \\
\hline \multicolumn{4}{|l|}{ Family history } \\
\hline COPD & 33 & 37 & 28 \\
\hline Lung cancer & $19^{*}$ & $11^{*}$ & $9 *$ \\
\hline \multicolumn{4}{|l|}{ Lung function } \\
\hline FEV 1 L & $1.86 \pm 0.48^{\star}$ & $1.25 \pm 0.48^{*}$ & $2.86 \pm 0.68^{*}$ \\
\hline FEV $1 \%$ pred & $73^{*}$ & $46^{*}$ & $99^{*}$ \\
\hline $\mathrm{FEV}_{1} / \mathrm{FVC}$ & $64 \pm 13^{*}$ & $46 \pm 8^{*}$ & $78 \pm 7^{*}$ \\
\hline Spirometric COPD ${ }^{\#}$ & $57^{*}$ & $100^{*}$ & $0^{*}$ \\
\hline
\end{tabular}

Data are presented as $\%$ or mean $\pm \mathrm{SD}$, unless otherwise stated. COPD: chronic obstructive pulmonary disease; FEV1: forced expiratory volume in one second; $\%$ pred: \% predicted; FVC: forced vital capacity. ${ }^{*}$ : according to Global Initiative for Chronic Obstructive Lung Disease stage 2. *: $p<0.05$ 
TABLE 3 Genotype frequencies for the nicotinic acetylcholine receptor a5 subunit single nucleotide polymorphism (rs16969968) in chronic obstructive pulmonary disease (COPD) and lung cancer (LC) cohorts compared with smoking controls

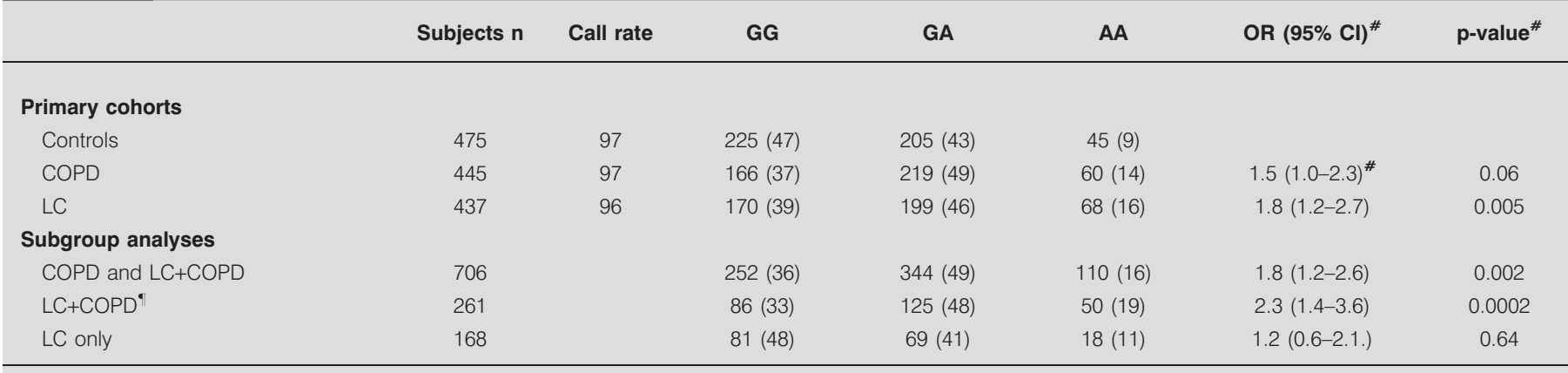

Data are presented as \% or $n(\%)$, unless otherwise stated. OR: odds ratio; $95 \% \mathrm{Cl}: 95 \%$ confidence interval. ${ }^{\#}$ : AA versus GA/GG compared with matched smoking controls (Mantel-Haenszel); ": AA versus GA/GG compared with COPD and lung cancer alone (OR 2.0, 95\% Cl 1.1-3.7; $p=0.02)$.

\section{Comparison with smoking controls}

The AA genotype of the rs16969968 SNP was not only found to be more frequent in lung cancer cases (16\%, OR 1.8, $\mathrm{p}=0.005)$ compared with controls $(9 \%)$ but WAS also more frequent in patients with COPD $(14 \%$, OR $1.5, \mathrm{p}=0.06$; tables 3 and 4). More importantly, when the lung cancer cases were divided according to spirometry results $(n=429$ meeting the previously mentioned criteria) into those with and without COPD (according to GOLD stage II+ criteria), the frequency of the AA genotype was 19\% (OR 2.3, p=0.0002) and 11\% (OR 1.2, $\mathrm{p}=0.64$ ) respectively (table 3 ). This observation shows that removing subjects with COPD from the lung cancer cohort weakens the nAChR SNP association (OR decreased from 1.8 to 1.2). Moreover, if the lung-cancer only cohort was tripled in size (from 168 to 504 cases), using the same genotype frequencies there is little difference statistically $(p=0.52)$ to suggest the result is not sensitive to differences in sample size (i.e. under-powering). In contrast, when the lung cancer plus COPD cohort (where COPD pre-dates the lung cancer) was added to the COPD cohort, the association between the AA genotype of the rs16969968 SNP and COPD compared with controls (16 versus $9 \%$ ) became statistically significant due to the larger sample size (OR 1.8, $\mathrm{p}=0.002)$. When the lung cancer association was re-examined by dividing lung cancer cases by reduced $\mathrm{FEV} 1 \%$ predicted ( $\leqslant 80$ versus $>81 \%$ pred) and by reduced ratio (FEV1/forced vital capacity $(\mathrm{FVC})<70$ versus $\geqslant 70 \%$ ) the same results were found (data not shown). The AA genotype frequency was also significantly higher in 261 patients with both lung cancer and COPD than in 445 patients with COPD only $(\mathrm{p}=0.05)$ or in 168 patients with lung cancer only $(p=0.02)$, although the numbers are small and failed to reach significance after correction for the number of comparisons using the Bonferonni approach. The present study found the association between the $\mathrm{nAChR}$ genotype in the lung cancer cohort was independent of cancer histology (data not shown) but linearly related to absolute FEV1 and FEV1\% pred (ANOVA $p=0.02$ and $p=0.001$, respectively, and test for linear trend $\mathrm{p}=0.03$ and $\mathrm{p}=0.001$, respectively).

\section{Comparison with stratification for COPD and lung cancer}

When the present authors stratified for COPD across all cohorts and compared those with no evidence of COPD (by combining 475 controls with 168 lung cancer only cases) with those with spirometric evidence of COPD (by combining 445 COPD patients with 261 cases of lung cancer plus COPD), the nAChR SNP was found to be significantly associated with COPD (OR 1.7, $p=0.002$ ). This contrasts with the results observed when the current authors stratified for lung cancer across all cohorts and compared those with no evidence of lung cancer (by combining 475 controls with 445 COPD patients) with 437 lung cancer cases and found that, although the nAChR SNP was associated with lung cancer, both the

TABLE 4 Genotype frequencies for the nicotinic acetylcholine receptor a5 subunit single nucleotide polymorphism (rs16969968) across all cohorts stratified by chronic obstructive pulmonary disease (COPD) and lung cancer (LC)

$\begin{array}{lllll}\text { Subjects n } & \text { GG } & \text { GA } & \text { AA } & \text { OR }(95 \% \text { Cl) }\end{array}$

Cohorts stratified by COPD

\begin{tabular}{|c|c|c|c|c|c|c|}
\hline No COPD & 643 & $306(48)$ & 274 (43) & $63(10)$ & & \\
\hline COPD & 706 & 252 (36) & 344 (49) & 110 (16) & $1.7(1.2-2.4)$ & 0.002 \\
\hline \multicolumn{7}{|c|}{ Cohorts stratified by LC } \\
\hline No LC" & 920 & 391 (43) & 424 (46) & $105(11)$ & & \\
\hline $\mathrm{LC}^{+}$ & 437 & $170(39)$ & $199(46)$ & $68(16)$ & $1.4(1.0-2.0)$ & 0.03 \\
\hline
\end{tabular}

Data are presented as n (\%), unless otherwise stated. OR: odds ratio; 95\% Cl: 95\% confidence interval. \#: AA versus GA/GG (Mantel-Haenszel); ${ }^{\prime}: 48 \%$ with no LC have COPD; ${ }^{+}: 60 \%$ with LC have COPD. 
magnitude and significance were less than when stratified by COPD (OR 1.4, $\mathrm{p}=0.03$ ). It is likely that this reflects a stronger association between the $\mathrm{nAChR}$ SNP and COPD than compared with lung cancer, where $60 \%$ of lung cancer cases also had COPD

\section{DISCUSSION}

The current findings suggest polymorphisms in the nAChR gene are associated with the presence of COPD and that the recently reported association with lung cancer is either confounded by coexisting COPD or additive with COPD. With respect to the latter, many studies have suggested that genetic factors underlying COPD, characterised by lung parenchymal destruction and small airway fibrosis, may be shared with lung cancer susceptibility [10, 21, 22]. This would seem plausible if these genetic effects confer an aberrant (or maladaptive) response to smoking- induced inflammatory effects underlying the development of COPD and the initiation of cancer. However, as reduced FEV1 (COPD) has also been independently associated with lung cancer [9], lung cancer mortality [12] and PAD [13], confounding by the presence of COPD could also explain these recently reported associations [1-3]. Importantly, previous studies suggest that this receptor could underlie the development of COPD [27-29]. Based on these observations, the present authors propose that the association of the $\alpha 5$ subunit nAChR SNP with lung cancer [1-3] and lung cancer mortality [2] could be largely explained through its relationship to COPD.

Failing to account for confounding variables is a fundamental consideration in epidemiological studies [30-32]. Regardless of the size of the study, confounding can lead to spurious conclusions. There is considerable epidemiological, clinical and in vitro data to suggest that the factors underlying lung cancer, apart from smoking, may also be relevant in COPD. Recent observations suggest that genetic factors could be shared by these two diseases [21, 22]. Prevalence studies have highlighted just how much COPD is under-diagnosed and therefore under-reported. When spirometry is used to screen smokers, estimates of undiagnosed COPD range 50-80\% [5, 15-17]. Therefore, it is understandable that in recruiting volunteer smokers, the prevalence of COPD based on spirometry could be $20-30 \%$, although many of these people would be unaware of their diagnosis. Equally, both retrospective and prospective studies have suggested that $\geqslant 50 \%$ of smokers with lung cancer have spirometric evidence of COPD at the time lung cancer is diagnosed [11, 18-21]. The current results are consistent with these findings. Large-scale epidemiological studies [1-3] do not routinely measure lung function in patients with lung cancer (or controls); however, the few that have, found that poor lung function correlates with a poor outcome in those subsequently diagnosed with lung cancer [12]. This highlights the need to control for the presence of COPD in these studies [1-3]. It is not clear whether (or by how much) the presence of lung cancer may alter lung function at the time of diagnosis of lung cancer. Clinically, it is recommended that lung function be performed in early stage lung cancer in order to assess peri-operative risk and the impact of surgical resection on post-operative lung function and respiratory reserve [33, 34]. Studies examining lung function after lobectomy suggest that lung function is only minimally affected [35]. In addition, in the prospective study by MANNINO et al. [11], where lung function was performed at baseline and prior to lung cancer diagnosis, the prevalence of GOLD stage I+ COPD was $48 \%$ in incident lung cancer cases, which is comparable to that reported in studies where lung function was performed at the time of diagnosis [18-20]. In the lung cancer cases in the present study, lung function data was obtained at the time of (or very soon after) diagnosis and in the absence of significant compromise from lung collapse and pleural effusions. Given these factors, it is believed that the effect of lung cancer on lung function in the current study is likely to be minimal and that this will not significantly impair the ability to divide the lung cancer cohort by those with and without COPD (or at least above and below diagnostic FEV1 or FEV1/FVC values). It can be concluded from the present study that just over $50 \%$ of the lung cancer cases have coexisting COPD, which is consistent with the literature [18-21]. Moreover, this supports the belief that COPD may confound the association between the nAChR gene cluster on chromosome 15q.25 and lung cancer. If this is correct, then any further associations reported from the GWAS may result from a similar confounding effect with COPD.

The association described herein between the nAChR gene cluster on chromosome 15q.25 and COPD has recently been confirmed in a GWAS of patients with COPD [36]. This region contains a number of genes that encode proteins which are subunits of the nAChR. Several studies support the current hypothesis that SNPs in this gene may have functional significance and confer susceptibility to COPD and, to a lesser degree, lung cancer [27-29]. This is because the nAChR is part of a non-neuronal cholinergic system, which is of considerable importance in the lung. Specifically, these receptors are expressed throughout the lung and mediate the direct effects of nicotine on the neurotransmitter acetylcholine. These receptors are expressed on bronchial epithelial cells and a number of inflammatory cells including mast cells, neutrophils, monocytes and lymphocytes [28, 29]. It has been proposed that under conditions of chronic nicotine exposure, the $\alpha 5$ subunit of the $\mathrm{nAChR}$ may be involved in modifying the inflammatory response from smoking. If this were true then it is plausible the SNP genotyped herein (rs 16969968) has functional affects as it results in a nonsynonomous amino acid change (aspartic acid to asparagine) in amino acid position 398 in the $\alpha 5$ subunit of the nAChR. This amino acid is found in the central part of the second intracellular loop and is highly conserved across species. Functional studies will be needed to confirm this hypothesis.

The current results, showing that the nAChR gene locus on chromosome $15 q 25$ is strongly associated with COPD, have been replicated in a GWAS of two other COPD cohorts [36]. In the present study, the genotype frequencies for the $\alpha 5 \mathrm{nAChR}$ G/A SNP (rs16969968) in lung cancer cases is linearly related to the FEV1 \% pred (ANOVA $\mathrm{p}=0.001$ and test for linear trend $p=0.001)$. These observations, together with current understanding of the possible functionality of the rs16969968 SNP in nicotine-induced airway inflammation, demonstrate that an important biological role for this SNP in the pathogenesis of COPD is very plausible. However, an overlapping but lesser role in lung cancer cannot be excluded. Based on this stratified analysis, the present authors argue that the nAChR effect is 
greater with COPD than with lung cancer and that this observation is not due to under-powering. Therefore, COPD is more likely to act as a confounder, as apposed to an effect modifier, in the recently reported associations between $\mathrm{nAChR}$ SNPs and lung cancer [1-3]. This is based on the following rationale. First, if COPD was an effect modifier rather than a confounder, one would expect to see a robust association between $\mathrm{nAChR}$ and lung cancer when COPD was excluded from the model (this was not evident, even after allowing for issues of power). Secondly, one would expect to see little, if any, association between COPD and $\mathrm{nAChR}$ when lung cancer is excluded (previous studies clearly show a robust association with COPD) [36]. Thirdly, when the lung cancer cohort was divided into those with and without COPD, a greater proportion of those with no COPD would be expected to carry the AA genotype compared with those with COPD (instead, the opposite was found with $75 \%$ of the lung cancer cases carrying the risk genotype having COPD). Finally, further support for the present conclusion, that the $\mathrm{nAChR}$ SNP is associated with COPD, originates from the surprising dose-dependent relationship between lung function (absolute FEV1 \% pred, FEV1 and FEV1/FVC) and the A allele of the rs16969968 SNP in the lung cancer cohort where recruitment was independent of lung function (data not shown, AA associated with the lowest lung function). Although data from the present study are supportive of an important (most likely confounding) role for COPD, greater clarification of the relationship between nAChR SNPs, COPD and lung cancer will require larger studies to confirm the current interesting findings.

The present study outlines the important inter-relationship between COPD and lung cancer, particularly the potential role COPD has in confounding associations reported in casecontrol epidemiological studies of lung cancer. The current authors suggest that not only is the reported association between the chromosome $15 q 25$ region and lung cancer partly confounded by COPD but that COPD might also confound the reported association with peripheral arterial disease [13] and lung cancer mortality [1, 2, 12]. To the current authors' knowledge, this is the first time such a confounding effect has been identified in the current era of GWAS (excluding population admixture) but is unlikely to be the last. This suggests that well-designed candidate gene studies, where confounding variables can be measured and accounted for in matched or stratified analyses, still have utility in assessing the reliability of the genetic associations reported in the GWAS. In a recent review of GWAS, heterogeneity of control cohorts (to sufficiently power the study), misclassification of controls (due to a failure to screen them), and a failure to account for environmental factors (such as smoking) and confounding were all identified as potential limitations [37]. The current finding that the chromosome 15q25 locus has an important role in COPD has been replicated in a further two COPD cohorts [36], thereby identifying a new target for novel treatments [28]. In vitro studies suggest that genetic variation in the genes encoding the $\mathrm{nAChR}$ subunits could be directly involved in conferring susceptibility to COPD through aberrant response to smoking and airway inflammation. These observations do not exclude a possible role for this locus in lung cancer (or nicotine addiction); however, the current results suggest it is certainly of importance in COPD (i.e. triple whammy).
In conclusion, future genetic association studies of lung cancer will need to consider the potential confounding effects of chronic obstructive pulmonary disease by measuring lung function in study participants.

\section{REFERENCES}

1 Thorgeirsson TE, Geller F, Sulem P, et al. A variant associated with nicotine dependence, lung cancer and peripheral arterial disease. Nature 2008; 452: 638-642.

2 Hung RJ, McKay JD, Gaborieau V, et al. A susceptibility locus for lung cancer maps to nicotinic acetylcholine receptor subunit genes on 15q25. Nature 2008; 452: 633-637.

3 Amos CI, Wu X, Broderick P, et al. Genome-wide association scan of tag SNPs identifies a susceptibility locus for lung cancer at 15q25.1. Nat Genet 2008; 40: 616-622.

4 Saccone SF, Hinrichs AL, Saccone NL, et al. Cholinergic nicotinic receptor genes implicated in a nicotine dependence association study targeting 348 candidate genes with 3713 SNPs. Hum Mol Genet 2007; 16: 36-49.

5 Mannino DM, Watt G, Hole D, et al. The natural history of chronic obstructive pulmonary disease. Eur Respir J 2006; 27: 627-643.

6 Fletcher C, Peto R. The natural history of chronic airflow obstruction. Br Med J 1977; 1: 1645-1648.

7 Lokke A, Lange P, Scharling H, Fabricius P, Vestbo J. Developing COPD: a 25 year follow up study of the general population. Thorax 2006; 61: 935-939.

8 Burrows B, Knudson RJ, Cline MG, Lebowitz MD. Qualitative relationships between cigarette smoking and ventilatory function. Am Rev Respir Dis 1977; 115: 195-205.

9 Tockman MS, Anthonisen NR, Wright EC, Donithan MG. Airways obstruction and the risk for lung cancer. Ann Intern Med 1987; 106: 512-518.

10 Young RP, Hopkins RJ, Eaton TE. Forced expiratory volume in one second: not just a lung function test but a marker of premature death from all causes. Eur Respir J 2007; 30: 616-622.

11 Mannino DM, Aguayo SM, Petty TL, Redd SC. Low lung function and incident lung cancer in the United States: data from the First National Health and Nutrition Examination Survey Follow-up. Arch Int Med 2003; 163: 1475-1480.

12 Kuller LH, Ockene J, Meilahn E, Svendsen KH. Relation of forced expiratory volume in one second (FEV1) to lung cancer mortality in the Multiple Risk Factor Intervention Trial (MRFIT). Am J Epidemiol 1990; 132: 265-274.

13 Engstrom G, Hedblad B, Valind S, Janzon L. Asymptomatic leg and carotid atherosclerosis in smokers is related to degree of ventilatory capacity: longitudinal and cross-sectional results from "Men born in 1914", Sweden. Atherosclerosis 2001; 155: 237-243.

14 Anthonisen NR, Connett JE, Murray RP. Smoking and lung function of Lung Health Study participants after 11 years. Am J Respir Crit Care Med 2002; 166: 675-679.

15 Lindberg A, Bjerg-Backlund A, Ronmark E, Larsson LG, Lundback B. Prevalence and under-diagnosis of COPD by disease severity and attributable fraction of smoking. Report from the Obstructive Lung Disease in Northern Sweden Studies. Respir Med 2006; 100: 264-272. 
16 Stav D, Raz M. Prevalence of chronic obstructive pulmonary disease among smokers aged 45 and up in Israel. Isr Med Assoc J 2007; 9: 800-802.

17 Zaas D, Wise R, Wiener C, Longcope Spirometry Investigation Team, Airways obstruction is common but unsuspected in patients admitted to a general medicine service. Chest 2004; 125: 106-111.

18 Loganathan RS, Stover DE, Shi W, Venkatraman E. Prevalence of COPD in women compared to men around the time of diagnosis of primary lung cancer. Chest 2006; 129: 1305-1312.

19 Lopez-Encuentra A, Bronchogenic Carcinoma Co-operative Group, Comorbidity in operable lung cancer: a multicenter descriptive study on 2992 patients. Lung Cancer 2002; 35: 263-269.

20 Congleton J, Muers MF. The incidence of airflow obstruction in bronchial carcinoma, its relation to breathlessness, and response to bronchodilator therapy. Respir Med 1995; 89: 291-296.

21 Young RP, Hopkins RJ, Gamble GD, et al. A gene based risk score identifies smokers and ex-smokers at high risk of lung cancer. Respirology 2008; 13: Suppl. 2, TP143.

22 Brody JS, Spira A. Chronic obstructive pulmonary disease, inflammation, and lung cancer. Proc Am Thorac Soc 2006; 3: 535-538.

23 Moskvina V, Holmans $\mathrm{P}$, Schmidt KM, Craddock N. Design of case-controls studies with unscreened controls. Ann Hum Genet 2005; 69: 566-576.

24 Pritchard JK, Stephens M, Donnelly P. Inference of population structure from multi-locus genotype data. Genetics 2000; 155: 945-959.

25 Yang P, Allen MS, Aubry MC, et al. Clinical features of 5,628 primary lung cancer patients: experience at Mayo Clinic from 1997 to 2003. Chest 2005; 128: 452-462.

26 International Hapmap Project. www.hapmap.org Date last accessed: August 2, 2008. Date last updated: July 1, 2008.
27 Profita M, Giorgi RD, Sala A, et al. Muscarinic receptors, leukotriene $\mathrm{B}_{4}$ production and neutrophilic inflammation in COPD patients. Allergy 2005; 60: 1361-1369.

28 Gwilt CR, Donnelly LE, Rogers DF. The non-neuronal cholinergic system in the airways: An unappreciated regulatory role in pulmonary inflammation? Pharmacol Ther 2007; 115: 208-222.

29 Carlisle DL, Hopkins TM, Gaither-Davis A, et al. Nicotine signals through muscle-type and neuronal nicotinic acetylcholine receptors in both human bronchial epithelial cells and airway fibrosis. Respir Res 2004; 5: 27-42.

30 Khoury MJ, Yang Q. The future of genetic studies of complex human diseases: an epidemiologic perspective. Epidemiology 1998; 9: 350-354.

31 Campbell H, Rudan I. Interpretation of genetic association studies in complex disease. Pharmacogenetics J 2002; 6: 349-360.

32 Amos CI. Successful design and conduct of genome-wide association studies. Hum Mol Genet 2007; 16: 220-225.

33 Zeiher BG, Gross TJ, Kern JA, Lanza LA, Peterson MW. Predicting postoperative pulmonary function in patients undergoing lung resection. Chest 1995; 108: 68-72.

34 Win T, Jackson A, Sharples L, et al. Relationship between pulmonary function and lung cancer surgical outcome. Eur Respir J 2005; 25: 594-599.

35 Baldi S, Ruffini E, Harari S, et al. Does lobectomy for lung cancer in patients with chronic obstructive pulmonary disease affect lung function? A multicentre national study. J Thorac Cardiovasc Surgery 2005; 130: 1616-1622.

36 Pillai SG, Shianna K, Ge D, et al. Genome-wide association study of chronic obstructive pulmonary disease (COPD) in a case control population from Norway. American Thoracic Society, Toronto. Am J Respir Crit Care Med 2008; 177: A776.

37 Pearson TA, Manolio TA. How to interpret a genome-wide association study. JAMA 2008; 299: 1335-1344. 